\title{
Examining the use of radiation therapy for cholangiocarcinoma: benefits through modern techniques
}

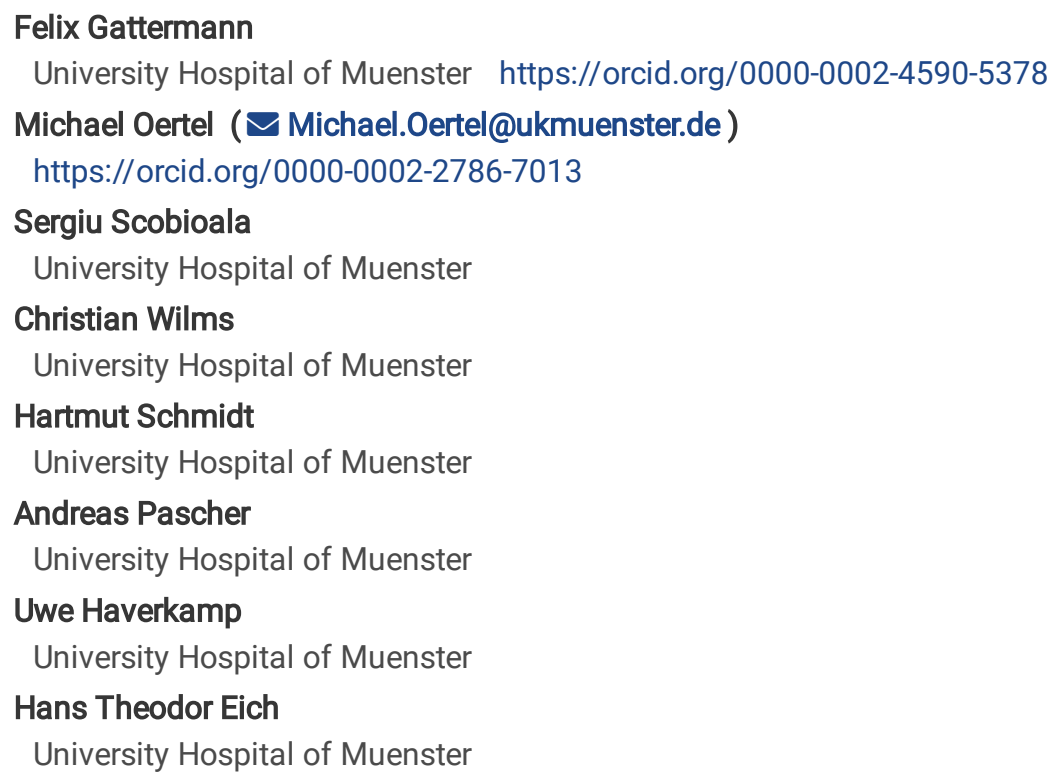

\section{Research}

Keywords: Cholangiocarcinoma, Bile duct cancer, Radiotherapy, Prognostic factors, Toxicities, Survival

Posted Date: August 18th, 2020

DOI: https://doi.org/10.21203/rs.3.rs-58656/v1

License: (c) (i) This work is licensed under a Creative Commons Attribution 4.0 International License. Read Full License 


\section{Abstract}

\section{Background}

Cholangiocarcinoma (CCA) is a rare malignant tumor of the bile duct epithelium. At first diagnosis, only a minority of patients is eligible for surgery, which is regarded as the only curative treatment. This study examines the role of radiation therapy (RT) and chemoradiotherapy (CRT) in the definitive and adjuvant treatment situation.

\section{Methods}

The monocentric retrospective analysis included 39 patients (31 males, 8 females) with CCA undergoing 53 RT series. Data was collected from January 2005 to September 2018. There were 11 cases of CRT, 6 of which were definitive. Surgery was either palliative $(n=6)$ or radical $(n=15)$.

\section{Results}

After RT, median overall survival (OS) was 10.4 months (mo; 95\% confidence interval [Cl] 6.6-14.2), median progression-free survival (PFS) was $5.6 \mathrm{mo}(95 \% \mathrm{Cl} 3-8.2)$, median duration of local control (DOLC) was $8.9 \mathrm{mo}(95 \% \mathrm{Cl}$ 4.7-13.1) and 1-year OS rate was $44.7 \%$. There was a significant difference between patients with and without locoregional lymph node metastasis (OS: 4.3 mo vs. 15.4 mo, $p=0.031$; PFS: 2.1 mo vs. $11.5 \mathrm{mo}, \mathrm{p}<0.0005$; DOLC: $4.2 \mathrm{mo}$ vs. $12.3 \mathrm{mo}, \mathrm{p}=0.02$ ). After treatment of a primary tumor, DOLC was about twice as long as in the recurrent situation ( $10.4 \mathrm{mo}$ vs. $5.4 \mathrm{mo}, \mathrm{p}=0.032$ ). Conservative therapy significantly elevated the risk of local recurrence compared to radical surgery in univariate $(H R 11.04, p=0.004)$ and multivariate (HR 98.34, $p=0.024)$ analysis. Tomotherapy may be advantageous with respect to local recurrence and survival.

Side effects were mostly classified as grade I-II according to CTCAE. There were 10 toxicities of grade III and 4 of grade IV, all affecting blood parameters. Termination of RT and increased glutamic pyruvic transaminase (GPT) were significantly less frequent after stereotactic body radiation therapy and hypofractionation.

\section{Conclusion}

RT can achieve local control in patients with CCA. However, since overall prognosis remains poor, effective combination therapies are needed. Toxicities of RT are manageable but require close clinical and laboratory follow-up.

\section{Introduction}

Cholangiocarcinoma (CCA) is a rare malignant tumor of the bile duct epithelium with an annual incidence between 0.35 and 2 per 100,000 in the Western world $(1,2)$. It may be categorized as intrahepatic, extrahepatic or hilar, the latter being further subdivided by the BismuthCorlette classification (3).

Surgery is regarded as the only curative treatment $(4,5)$, but prognosis remains dismal with a median OS of just 9 months (mo) without adjuvant treatment (6). Highly selected patients may be suitable for neoadjuvant chemoradiotherapy (CRT) followed by liver transplantation, which proved to be superior in respect of recurrence-free and overall survival $(\mathrm{OS})(7,8)$. However, only a minority of patients with CCA are eligible for surgery at first diagnosis, as symptoms usually do not occur until the disease is in an advanced stage. Additionally, CCA reveals an aggressive behavior with up to $60 \%$ local recurrences after curative resection (1), calling for additional treatments.

Brachytherapy $(1,9,10)$, photodynamic therapy $(11,12)$ and transarterial chemoembolization $(13,14)$ have been investigated without achieving convincing results, while causing potentially severe toxicities. Until now, the use of molecular targeted therapies and immunotherapy has not resulted in a substantial survival benefit $(1,15,16)$.

External beam radiotherapy (RT) can be used in the definitive or adjuvant treatment setting, ameliorating survival significantly $(1,4,17,18)$. Data from the Surveillance, Epidemiology, and End Results (SEER) database demonstrate an improvement of cancer specific survival for definitive RT (19). Furthermore, a study including patients with unresectable intrahepatic CCA from the National Cancer Data Base (NCDB) found an advantage of CRT compared to chemotherapy alone in the definitive treatment situation (20). In the postoperative setting, a prospective clinical trial investigating CRT yielded a promising median OS of $35 \mathrm{mo}$ (21). Similarly, adjuvant RT enhances median OS compared to surgery alone (6) and lowers the risk of death (22). In contrast, another study suggested a survival advantage for adjuvant RT only in the first 14 mo after total resection and the first 21 mo after subtotal resection (23). 
Overall, the role of RT is not well defined in the context of CCA, demanding further analysis. Due to the low incidence of CCA, prospective trials assessing the impact of RT are sparse and there is limited evidence for choosing a specific dose, technique or fractionation scheme. Consequently, we aimed to provide further insight into the role of RT in the treatment of patients with CCA and to identify prognostic factors.

\section{Methods}

\section{Patients}

This monocentric and retrospective analysis included 39 patients with intrahepatic $(n=11)$, hilar $(n=26)$ or both intra- and extrahepatic ( $n$ $=2$ ) CCA showing a total of 53 lesions treated at our institution from January 2005 to September 2018. Patient characteristics are shown in Table 1. Median age was 68 years both at first diagnosis (range: $40-83$ years) and at the beginning of RT (range: $41-83$ years). 
Table 1

Patient characteristics

\begin{tabular}{|c|c|c|}
\hline Characteristic & $\mathbf{n}$ & Percentage \\
\hline Males & 31 & 79.5 \\
\hline Females & 8 & 20.5 \\
\hline Primary tumor & 34 & 87.2 \\
\hline Relapse & 5 & 12.8 \\
\hline Intrahepatic & 11 & 28.2 \\
\hline Hilar & 26 & 66.7 \\
\hline Bismuth I & 4 & 10.3 \\
\hline Bismuth II & 2 & 5.1 \\
\hline Bismuth III & 8 & 20.5 \\
\hline Bismuth IV & 10 & 25.6 \\
\hline Bismuth unknown & 2 & 5.1 \\
\hline Intra- + extrahepatic & 2 & 5.1 \\
\hline UICC I & 1 & 2.6 \\
\hline UICC II & 2 & 5.1 \\
\hline UICC III & 6 & 15.4 \\
\hline UICC IV & 14 & 35.9 \\
\hline UICC unknown & 16 & 41 \\
\hline $\mathrm{T} 1$ & 4 & 10.3 \\
\hline $\mathrm{T} 2$ & 14 & 35.9 \\
\hline T3 & 6 & 15.4 \\
\hline $\mathrm{T} 4$ & 2 & 5.1 \\
\hline T unknown & 13 & 33.3 \\
\hline No & 14 & 35.9 \\
\hline N1 & 11 & 28.2 \\
\hline N unknown & 14 & 35.9 \\
\hline MO & 13 & 33.3 \\
\hline M1 & 11 & 28.2 \\
\hline M unknown & 15 & 38.5 \\
\hline G1 & 1 & 2.6 \\
\hline G1-G2 & 1 & 2.6 \\
\hline $\mathrm{G} 2$ & 20 & 51.3 \\
\hline G2-G3 & 2 & 5.1 \\
\hline G3 & 7 & 17.9 \\
\hline G unknown & 8 & 20.5 \\
\hline
\end{tabular}


Data was collected from the department's clinical files and data management systems. Follow-up included regular clinical and imaging examinations and was performed in intervals of 3 mo for the first 2 years and every 6-12 mo afterwards. Mean duration of follow-up after the last day of RT was 13.6 mo. If patients were lost to follow-up, family physicians were contacted to gather information about treatment toxicities, local relapse or date of death.

\section{Treatments}

All 53 CCA lesions were treated with RT, either in the definitive or adjuvant setting. Median time from first diagnosis to start of RT was 4 mo. If possible, surgery was radical $(n=15)$, but in some cases only palliative $(n=6)$ intervention was feasible and 17 patients were inoperable (Table 2). Total tumor resection (R0 resection) was achieved in 3 patients. Eleven patients received chemotherapy with gemcitabine, either as monotherapy or in combination with a platinum agent. CRT was adjuvant in 5 cases and definitive in 6 cases. Most RT series were targeted at primary tumors $(n=34)$, followed by metastases $(n=12)$ and recurrences $(n=7)$. 
Table 2

Treatment characteristics

\begin{tabular}{|c|c|c|}
\hline Characteristic & $n$ & Percentage \\
\hline Across all 39 patients & 6 & 15.4 \\
\hline Palliative surgery & 15 & 38.5 \\
\hline Radical surgery & 17 & 43.6 \\
\hline Inoperable & 1 & 2.6 \\
\hline Surgery unknown & 3 & 7.7 \\
\hline Ro & 6 & 15.4 \\
\hline R1 & 7 & 17.9 \\
\hline R2 & 3 & 7.7 \\
\hline R unknown & 11 & 28.2 \\
\hline Chemotherapy & 25 & 64.1 \\
\hline No chemotherapy & 3 & 7.7 \\
\hline Chemotherapy unknown & 34 & 64.2 \\
\hline Across all 53 RT series & 7 & 13.2 \\
\hline RT of primary tumor & 12 & 22.6 \\
\hline RT of recurrence & 24 & 45.3 \\
\hline RT of metastasis & 7 & 13.2 \\
\hline Technique & 9 & 17 \\
\hline Tomotherapy & 12 & 22.6 \\
\hline SW IMRT & 1 & 1.9 \\
\hline S\&S IMRT & 4 & 7.5 \\
\hline VMAT & 49 & 92.5 \\
\hline 3D CRT & 36 & 67.9 \\
\hline Boost & 17 & 32.1 \\
\hline No Boost & 13 & 24.5 \\
\hline Fractionation & 40 & 75.5 \\
\hline \multicolumn{3}{|l|}{ SBRT } \\
\hline \multicolumn{3}{|l|}{ No SBRT } \\
\hline \multicolumn{3}{|l|}{ Normofractionated } \\
\hline \multicolumn{3}{|l|}{ Hypofractionated } \\
\hline
\end{tabular}

Regarding RT, the majority ( $n=36$ ) was treated with stereotactic body RT (SBRT). In these cases, target volume was covered by the $65 \%$ isodose. Treatment series were carried out as helical tomotherapy $(n=24)$, sliding window $(S W)$ intensity-modulated radiation therapy (IMRT, $n=7)$, step and shoot (S\&S) IMRT ( $n=9)$, volumetric modulated arc therapy (VMAT, $n=12)$ and three-dimensional conformal RT (3D $\mathrm{CRT}, \mathrm{n}=1)$. 
Hypofractionation $(n=40)$ encompassed treatment doses of 21-45 Gray in fractions of 3-12.5 Gray, whereas normofractionated ( $n=13$ ) treatments varied between 14 and 55.8 Gray in fractions of 1.8-2 Gray (Table 3).

The most frequently applied treatment regimens were 5 fractions of 7 Gray ( $n=19$; biologically effective dose (BED): 59.5 Gray, equivalent dose $\left(E Q D_{2}\right): 49.58$ Gray) and 3 fractions of 12.5 Gray (n=12; BED: 84.4 Gray, $E Q D_{2}: 70.31$ Gray).

Table 3

Treatment characteristics across all 53

RT series

\begin{tabular}{|lll|}
\hline Characteristic & \multicolumn{2}{l|}{ MedianRange } \\
\hline Fractions & 5 & $3-30$ \\
Radiation dose & 35 & $14-55.8$ \\
Fraction dose & 7 & $1.8-12.5$ \\
Liver dose & 10.4 & $0-58.6$ \\
$\begin{array}{l}\text { Stomach dose } \\
\text { Myelon dose }\end{array}$ & 1.5 & $0.1-56.1$ \\
$\begin{array}{l}\text { Right kidney } \\
\text { dose }\end{array}$ & 1.6 & $0-56.8$ \\
$\begin{array}{l}\text { Left kidney } \\
\text { dose }\end{array}$ & 1.5 & $0-27.1$ \\
\hline & & \\
\hline
\end{tabular}

\section{Statistical analysis}

Statistical analyses were performed using the software SPSS ${ }^{\circledR}$ version 24.0 (IBM ${ }^{\square}$, Armok, USA) and differences with a p-value below 0.05 were considered statistically significant. Time-dependent event curves were created by the Kaplan-Meier method and different curves dependent on a categorical variable were compared by means of the log-rank test. The duration of OS, local control (DOLC) and progression-free survival (PFS) was calculated from the first day of RT to the respective event. If a patient underwent RT several times due to progressive disease, he or she was included only once in the calculation of OS, PFS, DOLC and the corresponding Kaplan-Meier plots. In order to determine whether a local relapse had occurred in the planning target volume (PTV), plan and follow-up images before and after RT were analyzed.

The Cox proportional hazards model was applied to assess the impact of non-categorial variables on the occurrence of death, metastases or local relapse and to perform a multivariate analysis into which variables with a p-value below 0.1 according to univariate analysis were entered.

Fisher's exact test was applied to analyze the relationship between 2 categorical variables like treatment characteristics and toxicities. The latter were graded according to the Common Terminology Criteria for Adverse Events (CTCAE) (24).

\section{Results}

\section{Outcome}

At the end of follow-up, 31 of 39 patients (79.5\%) were deceased. Eight patients (20.5\%) were alive, of whom one (2.6\%) had a local recurrence and another one had a distant metastasis after RT. Overall, 19 patients (48.7\%) had at least one documented metastasis following RT. There were 10 hepatic, 7 lymph node, 5 pulmonary and 2 bone metastases. In addition, 6 cases of peritoneal carcinomatosis and a single case of spinal cord involvement were registered. Local recurrence was noted after 10 of 53 RT series (18.9\%).

In the entire cohort, 1-year local control rate (LC), 2-year LC and 1-year OS were $69.7 \%, 54.9 \%$ and $44.7 \%$, respectively. Median OS was 10.4 mo (95\% Cl 6.6-14.2; Table 4, Fig. 1). 


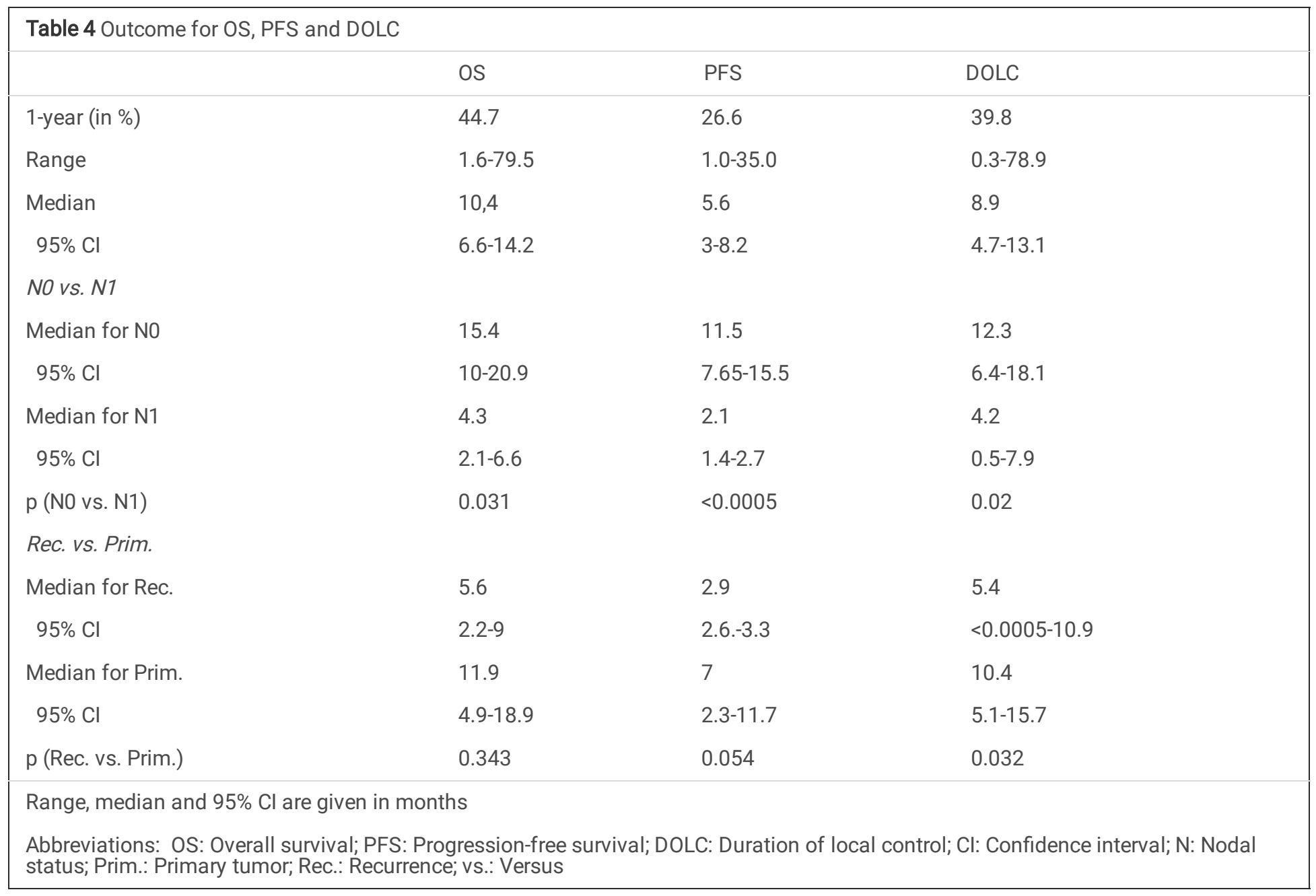

Adjuvant chemotherapy prolonged median OS from 9.8 to $18.8 \mathrm{mo}$, without statistical significance $(p=0.257)$. Similarly, there was a trend towards longer survival in non-metastatic patients $(12.3 \mathrm{mo}$ vs. $6.9 \mathrm{mo}, \mathrm{p}=0.485)$. After treatment of a primary tumor, median OS, PFS and DOLC were about twice as long as after treatment of a recurrent tumor (11.9 mo vs. 5.6 mo, 7 mo vs. 2.9 mo and 10.4 mo vs. 5.4 m), which was significant for median DOLC ( $p=0.032$; Table 4$)$. R-classification after surgery and ESRT did not have a significant impact on survival or local control. However, locoregional lymph node metastasis prior to RT resulted in a significantly worse outcome (4.3 mo vs. $15.4 \mathrm{mo}, \mathrm{p}$ $=0.031 ; 2.1$ mo vs. $11.5 \mathrm{mo}, p<0.0005$ and 4.2 mo vs. $12.3 \mathrm{mo}, p=0.02$ for OS, PFS and DOLC, respectively; Table 4, Fig. 2).

Locoregional lymph node involvement was also associated with a worse prognosis according to the Cox proportional hazards model (Table 5), both in univariate analysis (HR 2.9, $p=0.022$; HR 5.39, $p=0.001$; HR 3.29, $p=0.011 ;$ HR 12.25, $p=0.003$ for OS, PFS, DOLC and LC) and multivariate analysis (HR 2.33, $p=0.146$; HR 5.39, $p=0.001$; HR 2.1, $p=0.206$ for OS, PFS and DOLC). There was a tendency for higher T stages to have an unfavorable impact on OS in univariate analysis (HR 6.07 for T4 vs. T1, p = 0.051), which did not persist in multivariate analysis.

Conservative therapy was associated with significantly higher risk of local recurrence compared to radical surgery in univariate (HR 11.04 , $p=0.004)$ and multivariate (HR 98.34, $p=0.024)$ analysis. A similar effect was seen in terms of DOLC, which was significant in multivariate analysis (HR 3.72, $p=0.04)$. Similarly, palliative surgery was associated with a significantly higher risk of death than radical surgery (HR 6.04, $p=0.016)$ according to multivariate analysis. Interestingly, a palliative surgical procedure was associated with a higher risk of local relapse or death than no surgery at all (multivariate analysis: HR $5.81, p=0.019$ vs. $3.72, p=0.04$ ).

Table 5 Cox proportional hazards model 


\begin{tabular}{|c|c|c|c|c|c|c|c|c|c|c|c|c|}
\hline & \multicolumn{2}{|l|}{$\begin{array}{l}\text { Death } \\
\text { (OS) }\end{array}$} & \multicolumn{3}{|c|}{$\begin{array}{l}\text { Progression/Death } \\
\text { (PFS) }\end{array}$} & \multicolumn{4}{|c|}{ Local recurrence/Death (DOLC) } & \multicolumn{2}{|c|}{ Local recurrence (LC) } & \multirow[b]{2}{*}{$\mathrm{p}$} \\
\hline & $\mathrm{HR}$ & $95 \% \mathrm{Cl}$ & $\mathrm{p}$ & $\mathrm{HR}$ & $\begin{array}{l}95 \% \\
\mathrm{Cl}\end{array}$ & $\mathrm{p}$ & $\mathrm{HR}$ & $95 \% \mathrm{Cl}$ & $\mathrm{p}$ & $\mathrm{HR}$ & $95 \% \mathrm{Cl}$ & \\
\hline \multicolumn{13}{|l|}{ Univariate } \\
\hline $\begin{array}{l}\text { Rec. vs. } \\
\text { Prim. }\end{array}$ & 1.7 & $\begin{array}{l}0.57- \\
4.87\end{array}$ & 0.348 & 2.7 & $\begin{array}{l}0.95- \\
7.54\end{array}$ & 0.063 & 2.87 & $\begin{array}{l}1.05- \\
7.89\end{array}$ & 0.041 & 1.33 & $\begin{array}{l}0.33- \\
5.33\end{array}$ & 0.691 \\
\hline N1 vs. N0 & 2.9 & $1.17-7$ & 0.022 & 5.39 & $\begin{array}{l}2.07- \\
14.04\end{array}$ & 0.001 & 3.29 & $\begin{array}{l}1.32- \\
8.21\end{array}$ & 0.011 & 12.25 & $\begin{array}{l}2.34- \\
64.05\end{array}$ & 0.003 \\
\hline T4 vs. T1 & 6.07 & $1-37.1$ & 0.051 & 2.71 & $\begin{array}{l}0.48- \\
15.42\end{array}$ & 0.262 & 5.38 & $\begin{array}{l}0.88- \\
32.78\end{array}$ & 0.068 & $<0.0005$ & $<0.0005-$ & 0.994 \\
\hline $\begin{array}{l}\text { Inoperable } \\
\text { vs. Radical } \\
\text { surgery }\end{array}$ & 1.48 & $\begin{array}{l}0.66- \\
3.32\end{array}$ & 0.344 & 1.8 & $\begin{array}{l}0.82- \\
3.93\end{array}$ & 0.143 & 2.22 & $\begin{array}{l}0.97- \\
5.09\end{array}$ & 0.058 & 11.04 & $\begin{array}{l}2.2- \\
55.32\end{array}$ & 0.004 \\
\hline $\begin{array}{l}\text { Palliative } \\
\text { vs. Radical } \\
\text { surgery }\end{array}$ & 2.92 & $\begin{array}{l}0.87- \\
9.76\end{array}$ & 0.082 & 1.95 & $\begin{array}{l}0.61- \\
6.22\end{array}$ & 0.26 & 3.01 & $0.9-10.1$ & 0.075 & $<0.0005$ & $<0.0005-$ & 0.99 \\
\hline $\begin{array}{l}\text { SW IMRT } \\
\text { vs. Tomo }\end{array}$ & 3.6 & $\begin{array}{l}1.19- \\
10.66\end{array}$ & 0.023 & 4.01 & $\begin{array}{l}1.32- \\
12.17\end{array}$ & 0.014 & 2.6 & $\begin{array}{l}0.89- \\
7.58\end{array}$ & 0.082 & 4.85 & $\begin{array}{l}0.41- \\
56.91\end{array}$ & 0.208 \\
\hline \multirow{2}{*}{$\begin{array}{l}\text { VMAT vs. } \\
\text { Tomo }\end{array}$} & 2.9 & $\begin{array}{l}0.98- \\
8.59\end{array}$ & 0.055 & 2.22 & $\begin{array}{l}0.77- \\
6.39\end{array}$ & 0.138 & 2.83 & $0.95-8.5$ & 0.063 & 3.78 & $\begin{array}{l}0.31- \\
45.77\end{array}$ & 0.295 \\
\hline & 2.13 & \multirow{2}{*}{$\begin{array}{l}0.82- \\
5.53\end{array}$} & 0.122 & 1.26 & $0.5-$ & 0.629 & 1.67 & \multirow{2}{*}{$\begin{array}{l}0.65- \\
4.27\end{array}$} & 0.284 & 1.24 & \multirow{2}{*}{$\begin{array}{l}0.14- \\
10.7\end{array}$} & 0.848 \\
\hline vs. Tomo & 4.41 & & 0.17 & 1.84 & 3.15 & 0.564 & 0 & & 0.985 & 0 & & 0.996 \\
\hline $\begin{array}{l}\text { 3D CRT vs. } \\
\text { Tomo }\end{array}$ & & $\begin{array}{l}0.53- \\
36.79\end{array}$ & & & $\begin{array}{l}0.23- \\
14.6\end{array}$ & & & & & & $0-$. & \\
\hline \multicolumn{13}{|l|}{ Multivariate } \\
\hline $\begin{array}{l}\text { Rec. vs. } \\
\text { Prim. }\end{array}$ & 4.96 & $\begin{array}{l}1.27- \\
19.33\end{array}$ & 0.021 & 3.01 & $\begin{array}{l}0.86- \\
10.47\end{array}$ & 0.457 & 7.26 & $\begin{array}{l}1.91- \\
27.68\end{array}$ & 0.004 & 12.07 & $\begin{array}{l}0.66- \\
219.37\end{array}$ & 0.092 \\
\hline N1 vs. N0 & 2.33 & $\begin{array}{l}0.75- \\
7.27\end{array}$ & 0.146 & 5.39 & $\begin{array}{l}2.07- \\
14.04\end{array}$ & 0.001 & 2.1 & $\begin{array}{l}0.67- \\
6.65\end{array}$ & 0.206 & 0.88 & $\begin{array}{l}0.06- \\
12.6\end{array}$ & 0.922 \\
\hline T4 vs. T1 & 1.48 & $\begin{array}{l}0.07- \\
34.02\end{array}$ & 0.314 & 0.054 & $\begin{array}{l}0.003- \\
1.08\end{array}$ & 0.46 & 0.58 & $\begin{array}{l}0.03- \\
11.32\end{array}$ & 0.32 & 0.32 & $<0.0005$ & 1 \\
\hline $\begin{array}{l}\text { Inoperable } \\
\text { vs. Radical } \\
\text { surgery }\end{array}$ & 1.98 & $\begin{array}{l}0.55- \\
7.07\end{array}$ & 0.295 & 1.08 & $\begin{array}{l}0.387- \\
3.01\end{array}$ & 0.603 & 3.72 & $\begin{array}{l}1.06- \\
13.09\end{array}$ & 0.04 & 98.34 & $\begin{array}{l}1.8- \\
5339.03\end{array}$ & 0.024 \\
\hline $\begin{array}{l}\text { Palliative } \\
\text { vs. Radical } \\
\text { surgery }\end{array}$ & 6.04 & $\begin{array}{l}1.39- \\
26.24\end{array}$ & 0.016 & 2.18 & $\begin{array}{l}0.57- \\
8.33\end{array}$ & 0.132 & 5.81 & $\begin{array}{l}1.33- \\
25.38\end{array}$ & 0.019 & $<0.0005$ & $<0.0005-$ & 0.985 \\
\hline $\begin{array}{l}\text { SW IMRT } \\
\text { vs. Tomo }\end{array}$ & 4.21 & $\begin{array}{l}0.92- \\
19.29\end{array}$ & 0.064 & 4.28 & $\begin{array}{l}1.11- \\
16.5\end{array}$ & 0.189 & 4.01 & $\begin{array}{l}0.95- \\
17.12\end{array}$ & 0.059 & 87.9 & $\begin{array}{l}1.03- \\
7505.07\end{array}$ & 0.049 \\
\hline $\begin{array}{l}\text { VMAT vs. } \\
\text { Tomo }\end{array}$ & 9.66 & $\begin{array}{l}1.73- \\
53.87\end{array}$ & 0.01 & 3.79 & $\begin{array}{l}0.96- \\
14.9\end{array}$ & 0.196 & 6.98 & $\begin{array}{l}1.47- \\
33.13\end{array}$ & 0.015 & 13.46 & $\begin{array}{l}0.16- \\
1153.12\end{array}$ & 0.252 \\
\hline $\begin{array}{l}\text { S\&S IMRT } \\
\text { vs. Tomo }\end{array}$ & 5.81 & $\begin{array}{l}1.45- \\
23.31\end{array}$ & 0.013 & 4.71 & $\begin{array}{l}1.39- \\
16\end{array}$ & 0.748 & 5.84 & $\begin{array}{l}1.46- \\
23.39\end{array}$ & 0.013 & 75.15 & $\begin{array}{l}0.71- \\
7952.3\end{array}$ & 0.069 \\
\hline $\begin{array}{l}\text { 3D CRT vs. } \\
\text { Tomo }\end{array}$ & 21.78 & $\begin{array}{l}1.56- \\
303.66\end{array}$ & 0.022 & 4.43 & $\begin{array}{l}0.42- \\
46.51\end{array}$ & 0.66 & $<0.0005$ & $<0.0005$ & 0.989 & $<0.0005$ & $<0.0005-$ & 0.996 \\
\hline \multicolumn{13}{|c|}{ Median and $95 \% \mathrm{Cl}$ are given in months } \\
\hline \multicolumn{13}{|c|}{$\begin{array}{l}\text { Abbreviations: OS Overall survival; PFS Progression-free survival; DOLC Duration of local control; CI Confidence interval; Prim. Primary } \\
\text { tumor; Rec. Recurrence; vs. Versus; N Nodal status; T Tumor size; 3D CRT Three-dimensional conformal radiation therapy; SW Sliding } \\
\text { window; S\&S Step and shoot }\end{array}$} \\
\hline
\end{tabular}


Tomotherapy appeared to be a favorable prognostic factor regarding OS, PFS, DOLC and LC both in univariate and multivariate analysis (Table 5). In comparison with tomotherapy, the risk of death was significantly higher after VMAT (HR 9.66, $p=0.01$ ), S\&S IMRT (HR 5.81, $p$ $=0.013)$ and 3D CRT (HR 21.78, $p=0.022)$ in multivariate analysis. Furthermore, the risk of death or local recurrence after VMAT (HR 6.98, $p=0.015$ ) and S\&S IMRT (HR 5.84, $p=0.013$ ) was elevated compared to tomotherapy in multivariate analysis, similar to the risk of local recurrence after SW IMRT (HR 87.9, $p=0.049)$.

Neither total radiation dose nor dose per fraction had a significant impact on OS, PFS or DOLC in univariate Cox regression. Among those treated with normofractionated RT, an increase in the cumulative radiation dose was associated with a reduced mortality risk, almost at the level of statistical significance (HR 0.938, $p=0.066)$. The corresponding Pearson $(0.102)$ and Spearman $(0.116)$ correlation coefficients indicated no linear or monotonic relationship.

\section{Toxicities}

Acute toxicities were predominantly fatigue ( $4 x$ CTCAE grade I, $4 x$ grade II), stomach pain ( $6 x$ grade I, $2 x$ grade II), heartburn $(n=3)$ and nausea or emesis (10x grade I, 1x grade II). Three patients experienced intestinal colics. Moreover, fever indicating cholangitis, obstipation, diarrhea grade I and loss of appetite were reported by 2 patients each. Acute or chronic dermatological symptoms were not reported. Chronic side effects were rare and never exceeded CTCAE grade II during follow-up, with chronic fatigue being the most frequent $(n=7)$.

Nine patients had at least one acute side effect of grade III or IV. Altogether, 13 acute high-grade elevations ( $9 x$ grade III, $4 x$ grade IV) in liver parameters such as bilirubin $(n=5)$, alkaline phosphatase (AP, $n=3)$, gamma-glutamyltransferase $(\gamma-G T, n=1)$, glutamic pyruvic transaminase (GPT, $n=1)$ or glutamic oxaloacetic transaminase $(G O T, n=3)$ were registered. In addition, there was one case of leukopenia grade III. Often, laboratory values were already elevated prior to RT, which prompted us to calculate the pre- and post-RT difference. An acute increase by 3 CTCAE grades occurred only once, when elevated bilirubin worsened from grade I to grade IV. An increase by 2 grades was identified for GPT $(n=1)$, GOT $(n=4)$ and Y-GT $(n=3)$. When other laboratory values were affected, the CTCAE category always worsened by one grade compared to the pre-RT finding.

Higher-grade acute toxicities (grade III and IV) occurred in $11.1 \%$ of inoperable patients $(n=2)$. They were registered in $60 \%(n=3)$ and $22.2 \%(n=4)$ of patients after palliative and radical surgery, respectively. Nausea, the most common acute toxicity, was reported after $25 \%$ of definitive RT series and $40 \%$ of both palliative and radical surgical procedures. Chemotherapy had no significant impact on RT toxicity spectrum and severity.

Three patients did not complete RT due to side effects. One of them received RT after radical surgery and developed hemorrhage (type Forrest lla) from an inflamed anastomosis. Another patient refused to continue definitive RT after experiencing cholangitis with fever grade I, loss of appetite and laboratory values elevated to grade I or II. The third patient, undergoing RT after palliative surgery, also suffered from grade I fever and loss of appetite. In addition, he had leukocytopenia grade III and elevated liver parameters, i.e. bilirubin (grade III), AP (grade IV), GPT (grade III) and GOT (grade III).

According to Fisher's exact test, termination of RT ( $n=3 / 23.1 \%$ vs. $n=0, p=0.012)$ as well as an increase in GOT $(n=4 / 57.1 \%$ vs. $n=7 /$ $29.2 \%, p=0.042)$ and GPT ( $n=4 / 16 \%$ vs. $n=6 / 85.7 \%, p=0.001)$ were proportionately more frequent after normofractionated RT than hypofractionated RT. Similarly, significantly fewer cases of increased GPT ( $n=3 / 13.6 \%$ vs. $n=7 / 70 \%, p=0.003)$ and heartburn $(n=3 /$ $12.5 \%$ vs. $n=5 / 41.7 \%, p=0.044$ ) were registered following SBRT. There was also a tendency towards fewer high-grade side effects after SBRT and hypofractionation, without reaching statistical significance.

\section{Discussion}

The present study is one of the largest monocentric evaluations of RT for CCA. It identifies radical surgery, absence of locoregional lymph node metastasis, RT of a primary tumor and the use of tomotherapy as significant favorable prognostic factors. Furthermore, it adds new aspects to the current understanding of CCA treatment providing local control rates and a detailed survey of the toxicity spectrum which seems to be favorable after stereotactic body radiation therapy and hypofractionation.

Median OS was $10.4 \mathrm{mo}(95 \% \mathrm{Cl} 6.6-14.2)$ whereas the largest database analyses including 4,758 (6) and 2,842 (20) patients, respectively, found median OS to vary between 9 mo (definitive RT) (6), $13.6 \mathrm{mo}$ (definitive CRT) (20) and 16 mo (adjuvant RT) (6). Anyhow, comparability is limited due to pre-selected patient collectives. For example, eligibility criteria for the NCDB inquiry were curative intention of RT, absence of metastases and concomitant systemic therapy (20). Moreover, the recent Southwest Oncology Group (SWOG) S0809 trial, resulting in a median OS of $35 \mathrm{mo}$, only included patients with radical resection, no distant metastases and an Eastern Cooperative 
Oncology Group (ECOG) performance status of 0-1 allowing for sequential adjuvant chemotherapy followed by CRT (21). In that trial, 2year LC was $89 \%$ compared to $54.9 \%$ in our study, indicating a positive effect of radical surgical approaches and combined modality treatment on local control. In the large database analyses mentioned above, LC rates were not calculated. Two review articles estimated a pooled 1-year LC of $78.6 \%$ (4) and $83.4 \%$ (17), respectively, compared to $69.7 \%$ in our work. This difference may be attributable to the higher rate of SBRT $(100 \%(4,17)$ vs. $67.9 \%)$, enabling dose escalation. There is evidence for a positive dose-response relationship regarding RT for CCA $(25,26)$. In our study, SBRT prolonged median DOLC (8.9 mo vs. 5.6 mo), albeit not statistically significant $(p=$ 0.645).

Despite the dismal prognosis, one patient in our cohort who underwent radical hemihepatectomy followed by adjuvant chemotherapy and hypofractionated RT was still alive at the end of follow-up 6.6 years after initiation of RT, encouraging the use of combined modality treatment. Radical surgery remains the cornerstone of local therapy and constitutes a favorable prognostic factor $(1,4,6,17)$. Nevertheless, toxicities seem to occur more frequently after surgical intervention (see Results). Therefore, potential advantages and disadvantages of surgery have to be carefully considered, especially as our investigation found palliative surgery to be associated with poorer outcome in comparison with radical surgery and conservative treatment. A possible explanation may be an inadequate recovery time before initiation of RT. However, the median duration from palliative surgery to start of RT (76 days) was longer than in the radical surgery group (61 days), thus contradicting this hypothesis.

The apparent superiority of conservative treatment over palliative surgery may also be influenced by the more frequent use of chemotherapy in the non-surgical group $(n=6,37.5 \%$ vs. $n=1,16.7 \%)$. As mentioned in the results above, the addition of chemotherapy may prolong median OS (18.8 mo vs. $9.8 \mathrm{mo})$, although the difference was not statistically significant $(p=0.257)$ and other studies found a benefit of adjuvant chemotherapy only for patients with positive resection margins or locoregional lymph node metastasis $(5,27)$. Data regarding both nodal status and chemotherapy were available for 23 patients in our inquiry. In this sub-group, $20 \%$ of lymph-node positive patients received chemotherapy compared to $30 \%$ of those without. Furthermore, among the 25 patients for whom information regarding both nodal status and surgery was available, $45 \%$ of those with nodal involvement underwent radical surgery and $0 \%$ underwent a palliative procedure, compared to $64.3 \%$ and $21.4 \%$ of those without nodal involvement. Therefore, surgery and the use of chemotherapy might bias the prognostic impact of lymph node involvement.

Another potential bias may be an impaired performance status preventing the use of chemotherapy. Unfortunately, data concerning performance status were sparse, preventing definitive conclusions.

Previous studies showed an association between deterioration of recurrence-free survival and macrovascular invasion as well as tumor size $\geq 5 \mathrm{~cm}$ (28). A multivariate analysis based on data from the NCDB found advanced age (HR 1.01 per year, $p<0.001)$ and male gender (HR 1.13, $p=0.006$ ) to be predictors of a higher risk of death (20). Similar correlations were found in our study but did not reach statistical significance.

Helical tomotherapy might be superior to IMRT and 3D CRT regarding death and local recurrence (Table 5), due to its dose homogeneity and conformity even for irregularly shaped carcinomas. These features are enabled by the large number of beamlets resulting from 51 different beam directions per gantry rotation $(29,30)$. However, randomized controlled trials are needed to confirm potential advantages of this RT technique.

Chronic toxicities like fatigue may be influenced by the malignant disease itself with only a minor impact of RT (s. Toxicities). In spite of that, side effects after RT of CCA are common. According to a retrospective study including 96 patients undergoing SBRT, toxicities of CTCAE grade III or higher occurred significantly more often after treatment of CCA compared to hepatocellular carcinoma or hepatic metastases - probably because in CCA patients, the biliary tract itself is located within the target volume causing inflammation and edema, thereby increasing the risk of biliary obstruction (31).

In contrast to CCA, obstructing only small intrahepatic bile ducts, hilar Klatskin tumors may be even more likely to cause relevant bile stasis leading to high-grade biliary toxicities. According to a study in which $96.3 \%$ of the patients had a Klatskin tumor, $29.7 \%$ developed hyperbilirubinemia and cholangitis after SBRT (32). In the same study, high-grade duodenal or pyloric ulcers and even perforations reached $22.2 \%$, which may have been favored by the proximity of hilar tumors to the small intestine and stomach. Looking at our collective that included $66.7 \%$ Klatskin tumors, duodenal or gastric ulcers were not found and high-grade toxicities occurred in $16.1 \%$. The treatment of a patient cohort with $26.2 \%$ hilar CCA $(n=11)$ was accompanied by an even more favorable toxicity profile of $12 \%$ grade III toxicities and no grade IV toxicities in all RT series (33). 
Overall, SBRT is frequently associated with high-grade elevation of liver enzymes within the first 3 mo after initiation of RT (20\%-55.5\% (32, 34)). Correspondingly, $11.3 \%$ of our RT series and $8.3 \%$ of our SBRT treatments produced similar findings, with SBRT also featuring a lower rate of other toxicities (see Results). This may be explained by the steep dose gradients and narrow safety margins in SBRT, which delivers high doses to the tumor volume while sparing the surrounding tissue $(35,36)$. Tumor size may bias this finding, since hypofractionated doses and stereotactic approaches are usually applied for smaller tumors (36). In accordance, the median planning target volume (PTV) of our RT series was $39.5 \mathrm{~cm}^{3}$ for SBRT compared to $225.5 \mathrm{~cm}^{3}$ without SBRT. Being a hypofractionation regimen, SBRT requires fewer treatment days, which makes a combination with systemic therapies (e.g. SBRT between chemotherapy cycles) feasible and attractive.

Our study has limitations due to its monocentric and retrospective character. Together with the low incidence of CCA, this results in smaller patient numbers compared with analyses of large databases. Furthermore, the cause of death was unknown in some cases, so that several patients may not have died from their malignant disease. The duration of OS, PFS, DOLC and survival until the occurrence of local relapse was calculated from the first day of RT in each case, a method prone to lead-time bias. Moreover, as this study was meant to generate new hypotheses and not to prove a previously observed association, the significance level a was not adjusted although the influence of multiple variables was tested.

Ongoing and future clinical trials, like 2 prospective randomized phase III trials comparing adjuvant chemotherapy with CRT in extrahepatic CCA or gall bladder cancer (NCT02798510) and definitive chemotherapy with definitive CRT for unresectable CCA (NCT02773485), will hopefully shed more light on the role of RT for this malignancy.

\section{Conclusion}

RT has proven to be an effective therapy for CCA, providing good LC. Moreover, helical tomotherapy seems to be superior to other irradiation techniques regarding OS, DOLC and LC. Outcome deteriorates for patients with locoregional lymph node metastasis, in the recurrent treatment situation or with palliative surgery. Toxicities seem to be less likely after SBRT and are manageable but require close clinical as well as laboratory surveillance. Randomized clinical trials are necessary to improve combination therapies for CCA, which is still an orphan disease with poor prognosis.

\section{Abbreviations}

AP: Alkaline phosphatase; BED: Biologically effective dose; CCA: Cholangiocarcinoma; Cl: Confidence interval; CRT: Chemoradiotherapy; CTCAE: Common Terminology Criteria for Adverse Events; DOLC: Duration of local control; ECOG: Eastern Cooperative Oncology Group; $\mathrm{EQD}_{2}$ : Equivalent dose; G: Grading; GOT: Glutamic oxaloacetic transaminase; GPT: Glutamic pyruvic transaminase; HR: Hazard ratio; IMRT: Intensity-modulated radiation therapy; LC: Local control; M: Metastasis; n: Number; N: Nodal status; NCDB: National Cancer Data Base; OS: Overall survival; PFS: Progression-free survival; Prim.: Primary tumor; PTV: Planning target volume; R: Residual tumor; Rec.: Recurrence; RT: Radiotherapy; SBRT: Stereotactic body radiation therapy; SEER: Surveillance, S\&S Step and shoot; SW Sliding window; SWOG: Southwest Oncology Group Epidemiology, and End Results; T: Tumor size; VMAT: Volumetric modulated arc therapy; vs.: Versus; $Y$-GT: Gammaglutamyltransferase; 3D CRT: Three-dimensional conformal radiation therapy

\section{Declarations}

\section{Acknowledgements}

Not applicable

\section{Authors' contributions}

FG collected the data and performed the statistical analysis. The manuscript was written by FG and MO. MO, SS, CW, AP, HS, UH and HE contributed to the treatment of the patients included in this study. All authors read, corrected and approved the final manuscript.

\section{Funding}

Not applicable.

\section{Availability of data and materials}

The datasets used or analyzed during the current study are available from the corresponding author on reasonable request. 


\section{Ethics approval and consent to participate}

All procedures performed in studies involving human participants were in accordance with the ethical standards of the institutional and national research committee as well as with the 1964 Helsinki declaration including its later amendments or comparable ethical standards. This study has been approved by the local ethics committee (reference number 2018-669-f-S).

\section{Consent for publication}

Not applicable.

\section{Competing interests}

The authors declare that they have no competing interests.

\section{References}

1. Bridgewater JA, Goodman KA, Kalyan A, Mulcahy MF. Biliary Tract Cancer: Epidemiology, Radiotherapy, and Molecular Profiling. Am Soc Clin Oncol Educ Book. 2016;35:e194-203.

2. Saha SK, Zhu AX, Fuchs CS, Brooks GA. Forty-Year Trends in Cholangiocarcinoma Incidence in the U.S.: Intrahepatic Disease on the Rise. The Oncologist. 2016 Jan 5;21(5):594-9.

3. Bismuth H, Nakache R, Diamond T. Management strategies in resection for hilar cholangiocarcinoma. Ann Surg. 1992 Jan;215(1):318.

4. Lee J, Yoon WS, Koom WS, Rim CH. Efficacy of stereotactic body radiotherapy for unresectable or recurrent cholangiocarcinoma: a meta-analysis and systematic review. Strahlenther Onkol. 2018 Sep 11;

5. Brunner TB, Seufferlein T. Radiation therapy in cholangiocellular carcinomas. Best Pract Res Clin Gastroenterol. 2016 Aug;30(4):593602.

6. Shinohara ET, Mitra N, Guo M, Metz JM. Radiotherapy Is Associated With Improved Survival in Adjuvant and Palliative Treatment of Extrahepatic Cholangiocarcinomas. International Journal of Radiation Oncology*Biology*Physics. 2009 Jul 15;74(4):1191-8.

7. Rana A, Hong JC. Orthotopic liver transplantation in combination with neoadjuvant therapy: a new paradigm in the treatment of unresectable intrahepatic cholangiocarcinoma. Current Opinion in Gastroenterology. 2012 May;28(3):258-65.

8. Rea DJ, Heimbach JK, Rosen CB, Haddock MG, Alberts SR, Kremers WK, et al. Liver Transplantation with Neoadjuvant Chemoradiation is More Effective than Resection for Hilar Cholangiocarcinoma. Ann Surg. 2005 Sep;242(3):451-61.

9. Singh V, Kapoor R, Solanki KK, Singh G, Verma GR, Sharma SC. Endoscopic intraluminal brachytherapy and metal stent in malignant hilar biliary obstruction: a pilot study. Liver International. 2007;27(3):347-52.

10. Chen Y, Wang X-L, Yan Z-P, Cheng J-M, Wang J-H, Gong G-Q, et al. HDR-192Ir intraluminal brachytherapy in treatment of malignant obstructive jaundice. World J Gastroenterol. 2004 Dec 1;10(23):3506-10.

11. Witzigmann H, Berr F, Ringel U, Caca K, Uhlmann D, Schoppmeyer K, et al. Surgical and palliative management and outcome in 184 patients with hilar cholangiocarcinoma: palliative photodynamic therapy plus stenting is comparable to $\mathrm{r} 1 / \mathrm{r} 2 \mathrm{resection}$. Ann Surg. 2006 Aug;244(2):230-9.

12. Quyn AJ, Ziyaie D, Polignano FM, Tait IS. Photodynamic therapy is associated with an improvement in survival in patients with irresectable hilar cholangiocarcinoma. HPB (Oxford). 2009 Nov;11(7):570-7.

13. Llovet JM, Real MI, Montaña X, Planas R, Coll S, Aponte J, et al. Arterial embolisation or chemoembolisation versus symptomatic treatment in patients with unresectable hepatocellular carcinoma: a randomised controlled trial. Lancet. 2002 May 18;359(9319):1734-9.

14. Lo C-M, Ngan H, Tso W-K, Liu C-L, Lam C-M, Poon RT-P, et al. Randomized controlled trial of transarterial lipiodol chemoembolization for unresectable hepatocellular carcinoma. Hepatology. 2002 May;35(5):1164-71.

15. Valle JW, Lamarca A, Goyal L, Barriuso J, Zhu AX. REVIEW | New horizons for precision medicine in biliary tract cancers. Cancer Discov. 2017 Sep;7(9):943-62.

16. Tariq N-A, McNamara MG, Valle JW. Biliary tract cancers: current knowledge, clinical candidates and future challenges. Cancer Manag Res. 2019 Mar 29;11:2623-42.

17. Frakulli R, Buwenge M, Macchia G, Cammelli S, Deodato F, Cilla S, et al. Stereotactic body radiation therapy in cholangiocarcinoma: a systematic review. BJR. 2019 Feb 7;92(1097):20180688.

Page $13 / 16$ 
18. Sahai P, Kumar S. External radiotherapy and brachytherapy in the management of extrahepatic and intrahepatic cholangiocarcinoma: available evidence. Br J Radiol. 2017 Aug;90(1076):20170061.

19. Liu J, Zhong M, Feng Y, Zeng S, Wang Y, Xu H, et al. Prognostic Factors and Treatment Strategies for Intrahepatic Cholangiocarcinoma from 2004 to 2013: Population-Based SEER Analysis. Transl Oncol. 2019 Aug 22;12(11):1496-503.

20. Verma V, Kusi Appiah A, Lautenschlaeger T, Adeberg S, Simone CB, Lin C. Chemoradiotherapy versus chemotherapy alone for unresected intrahepatic cholangiocarcinoma: practice patterns and outcomes from the national cancer data base. $\mathrm{J}$ Gastrointest Oncol. 2018 Jun;9(3):527-35.

21. Ben-Josef E, Guthrie KA, El-Khoueiry AB, Corless CL, Zalupski MM, Lowy AM, et al. SWOG S0809: A Phase II Intergroup Trial of Adjuvant Capecitabine and Gemcitabine Followed by Radiotherapy and Concurrent Capecitabine in Extrahepatic Cholangiocarcinoma and Gallbladder Carcinoma. J Clin Oncol. 2015 Aug 20;33(24):2617-22.

22. Bonet Beltrán M, Allal AS, Gich I, Solé JM, Carrió I. Is adjuvant radiotherapy needed after curative resection of extrahepatic biliary tract cancers? A systematic review with a meta-analysis of observational studies. Cancer Treatment Reviews. 2012 Apr 1;38(2):111-9.

23. Fuller CD, Wang SJ, Choi M, Czito BG, Cornell J, Welzel TM, et al. Multimodality therapy for locoregional extrahepatic cholangiocarcinoma. Cancer. 2009;115(22):5175-83.

24. National Institutes of Health Common Terminology Criteria for Adverse Events (CTCAE) Version 4.0 Published: May 28, 2009 (v4.03: June 14, 2010), NIH Publication No. 09-5410 Revised June 2010, Reprinted June 2010. http://www.hrc.govt.nz/sites/default/files/CTCAE\%20manual\%20-\%20DMCC. pdf. Accessed 21 Jul 2019.

25. Tao R, Krishnan S, Bhosale PR, Javle MM, Aloia TA, Shroff RT, et al. Ablative Radiotherapy Doses Lead to a Substantial Prolongation of Survival in Patients With Inoperable Intrahepatic Cholangiocarcinoma: A Retrospective Dose Response Analysis. J Clin Oncol. 2016 Jan 20;34(3):219-26.

26. Crane CH, Macdonald KO, Vauthey JN, Yehuda P, Brown T, Curley S, et al. Limitations of conventional doses of chemoradiation for unresectable biliary cancer. Int J Radiat Oncol Biol Phys. 2002 Jul 15;53(4):969-74.

27. Cidon EU. Resectable Cholangiocarcinoma: Reviewing the Role of Adjuvant Strategies. Clin Med Insights Oncol. 2016;10:43-8.

28. Hyder O, Hatzaras I, Sotiropoulos GC, Paul A, Alexandrescu S, Marques H, et al. Recurrence after operative management of intrahepatic cholangiocarcinoma. Surgery. 2013 Jun;153(6):811-8.

29. Oliver M, Ansbacher W, Beckham WA. Comparing planning time, delivery time and plan quality for IMRT, RapidArc and tomotherapy. J Appl Clin Med Phys. 2009 Oct 7;10(4):117-31.

30. Zhu Z, Fu X. The radiation techniques of tomotherapy \& intensity-modulated radiation therapy applied to lung cancer. Transl Lung Cancer Res. 2015 Jun;4(3):265-74.

31. Osmundson EC, Wu Y, Luxton G, Bazan JG, Koong AC, Chang DT. Predictors of Toxicity Associated With Stereotactic Body Radiation Therapy to the Central Hepatobiliary Tract. International Journal of Radiation Oncology*Biology*Physics. 2015 Apr 1;91(5):986-94.

32. Kopek N, Holt MI, Hansen AT, Høyer M. Stereotactic body radiotherapy for unresectable cholangiocarcinoma. Radiotherapy and Oncology. 2010 Jan 1;94(1):47-52.

33. Mahadevan A, Dagoglu N, Mancias J, Raven K, Khwaja K, Tseng JF, et al. Stereotactic Body Radiotherapy (SBRT) for Intrahepatic and Hilar Cholangiocarcinoma. J Cancer. 2015 Aug 1;6(11):1099-104.

34. Tse RV, Hawkins M, Lockwood G, Kim JJ, Cummings B, Knox J, et al. Phase I Study of Individualized Stereotactic Body Radiotherapy for Hepatocellular Carcinoma and Intrahepatic Cholangiocarcinoma. JCO. 2008 Feb 1;26(4):657-64.

35. Herrmann H, Seppenwoolde Y, Georg D, Widder J. Image guidance: past and future of radiotherapy. Radiologe [Internet]. 2019 Jul 25 [cited 2019 Aug 25]; Available from: https://doi.org/10.1007/s00117-019-0573-y

36. Sterzing F, Brunner TB, Ernst I, Baus WW, Greve B, Herfarth K, et al. Stereotactic body radiotherapy for liver tumors: Principles and practical guidelines of the DEGRO Working Group on Stereotactic Radiotherapy. Strahlentherapie und Onkologie. 2014

Oct;190(10):872-81.

\section{Figures}



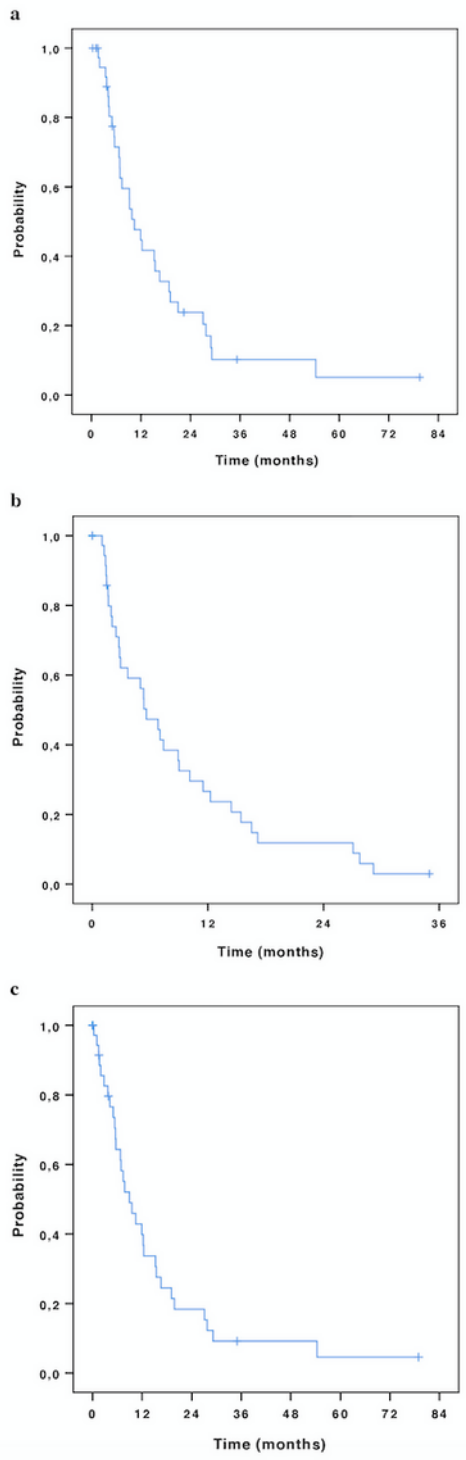

Figure 1

Kaplan-Meier plots for OS (a), PFS (b) and DOLC (c) 

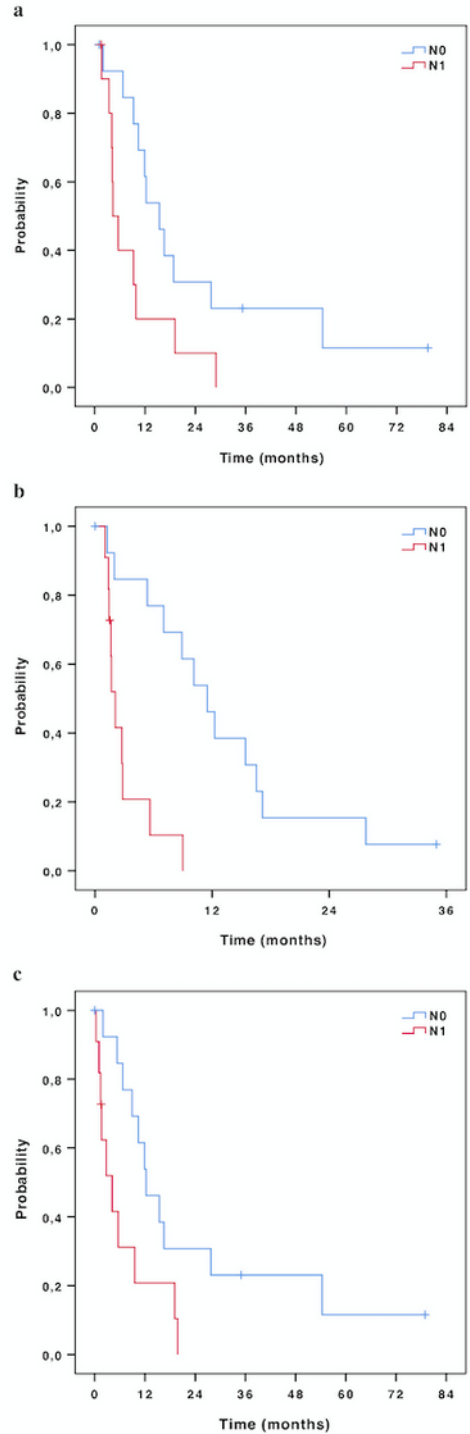

\section{Figure 2}

Prognostic value of nodal status: Kaplan-Meier plots showing a significant deterioration for lymph node positive patients (red) in comparison with lymph node negative patients (blue) regarding OS (a), PFS (b) and DOLC (c) 